\title{
The role of IL-15 and selenium in Graves ophthalmopathy
}

Se Jin Park and Jae II Shin

We read with great interest the News and Views article by Terry J. Smith and Raymond S. Douglas (Pharmacotherapy: Does selenium supplementation improve Graves ophthalmopathy? Nat. Rev. Endocrinol. 7, 505-506; 2011). ${ }^{1}$ The article discussed the study by Marcocci et al. ${ }^{2}$ which reported that selenium administration significantly improved quality of life, reduced ocular involvement and slowed progression of the disease in patients with mild Graves ophthalmopathy (GO) but did not explicate the exact pathomechanism. Smith and Douglas ${ }^{1}$ speculated that selenium might influence the sympathetic tone of the ocular muscles and, alternatively, it might reduce inflammation of the superior tarsal muscle of the eyelid. However, we would like to focus on a proinflammatory cytokine, IL-15, in the pathogenesis of GO associated with selenium deficiency.

Although not extensively studied yet, IL-15, which is involved in the differentiation of T-helper 17 cells, has been reported to be related to autoimmune thyroid diseases (AITD) such as Graves disease and Hashimoto thyroiditis. ${ }^{3}$ Higher concentrations of IL-15 were detected in sera from patients with Hashimoto thyroiditis compared with euthyroid individuals. ${ }^{4}$ Moreover, Pappa et al. ${ }^{5}$ detected IL-15 in 33\% of thyroid-associated ophthalmopathy biopsies from extraocular muscle.

Recently and importantly, Stazi and Trinti ${ }^{6}$ demonstrated that selenium deficiency and overexpression of IL-15 play a central role in the pathogenesis of AITD, by inhibiting apoptosis, leading to the perpetual inflammation and tissue destruction. Thyroid is especially sensitive to selenium deficiency because selenoproteins are significant in biosynthesis and activity of thyroid hormones and involved in inhibiting apoptosis. ${ }^{6}$ In addition, selenoproteins can modify thyrocytes function by acting as antioxidants and modifying redox status and thyroid hormone metabolism. ${ }^{7}$ In GO, not only deficiency of the essential trace mineral selenium but also overexpression of IL-15 may increase activation of effector mechanisms of epithelial damage by stimulating proliferation of T-helper 1 cells, cytokine production and intraepithelial lymphocyte cytotoxicity by protecting these lymphocytes from apoptosis. ${ }^{7}$

There is a strong association between thyroid-associated ophthalmopathy like GO and Graves disease in about $90 \%$ of patients and Hashimoto thyroiditis or a subclinical form of thyroid autoimmunity in the remaining $10 \%$ of patients with thyroid-associated ophthalmopathy, which suggests that these conditions share a common autoimmune pathogenesis. ${ }^{8-10}$

Therefore, it is possible that selenium deficiency is a formidable risk factor directly leading to thyroid damage through IL-15 overexpression in patients with AITD-like GO. Blocking IL-15 as well as selenium supplementation has the potential to provide a new therapeutic tool to prevent tissue damage of GO. However, further studies are necessary to elucidate the exact molecular role and pathway of IL-15 in the development of $\mathrm{GO}$ in the future.

Department of Pediatrics, Ajou University School of Medicine, Ajou University Hospital, Suwon, Korea (S. J. Park), The Institute of Kidney
Disease, Department of Pediatrics, Yonsei University College of Medicine, Severance Children's Hospital, Seoul, Korea (J. I. Shin). Correspondence to: J. I. Shin shinji@yuhs.ac

doi:10.1038/nrendo.2011.128-c1

Competing interest

The authors declare no competing interests.

1. Smith, T. J. \& Douglas, R. S. Pharmacotherapy: Does selenium supplementation improve Graves ophthalmopathy? Nat. Rev. Endocrinol. 7, 505-506 (2011).

2. Marcocci, C. et. al. Selenium and the course of mild Graves' orbitopathy. N. Engl. J. Med. 364, 1920-1931 (2011).

3. Figueroa-Vega, N. et.al. Increased circulating pro-inflammatory cytokines and Th17 lymphocytes in Hashimoto's thyroiditis. J. Clin. Endocrinol. Metab. 95, 953-962 (2010).

4. Simsek, G., Karter, Y., Aydin, S. \& Uzun, H. Osteoporotic cytokines and bone metabolism on rats with induced hyperthyroidism; changes as a result of reversal to euthyroidism. Chin. J. Physiol. 46, 181-186 (2003).

5. Pappa, A. et.al. Analysis of extraocular muscleinfiltrating T cells in thyroid-associated ophthalmopathy (TAO). Clin. Exp. Immunol. 109, 362-369 (1997).

6. Stazi, A. V. \& Trinti, B. Selenium status and over-expression of interleukin-15 in celiac disease and autoimmune thyroid diseases. Ann. Ist. Super. Sanita. 46, 389-399 (2010).

7. Stazi, A. V. \& Trinti, B. Selenium deficiency in celiac disease: risk of autoimmune thyroid diseases [in Italian]. Minerva Med. 99, 643-653 (2008).

8. Weetman, A. P. Thyroid-associated eye disease: pathophysiology. Lancet 338, 25-28 (1991).

9. Bahn, R. S. \& Heufelder, A. E. Pathogenesis of Graves' ophthalmopathy. N. Engl. J. Med. 329, 1468-1475 (1993).

10. Perros, P. \& Kendall-Taylor, P. Thyroid-associated ophthalmopathy: pathogenesis and clinical management. Baillieres. Clin. Endocrinol. Metab. 9, 115-135 (1995). 